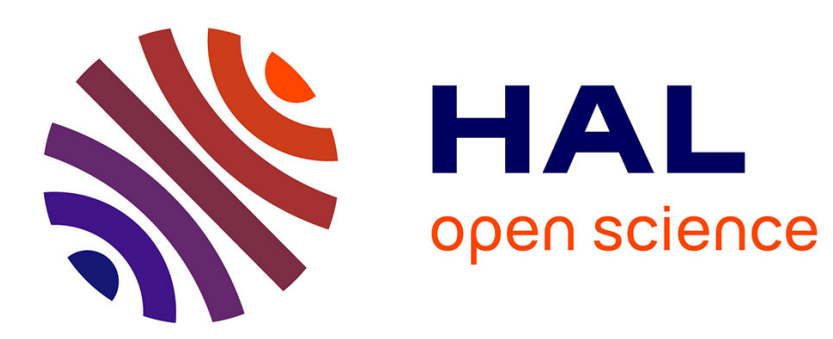

\title{
Random phase approximation correlation energies with exact Kohn-Sham exchange
}

Andreas Hesselmann, Andreas Goerling

\section{To cite this version:}

Andreas Hesselmann, Andreas Goerling. Random phase approximation correlation energies with exact Kohn-Sham exchange. Molecular Physics, 2010, 108 (03-04), pp.359-372. 10.1080/00268970903476662 . hal-00580676

\section{HAL Id: hal-00580676}

\section{https://hal.science/hal-00580676}

Submitted on 29 Mar 2011

HAL is a multi-disciplinary open access archive for the deposit and dissemination of scientific research documents, whether they are published or not. The documents may come from teaching and research institutions in France or abroad, or from public or private research centers.
L'archive ouverte pluridisciplinaire HAL, est destinée au dépôt et à la diffusion de documents scientifiques de niveau recherche, publiés ou non, émanant des établissements d'enseignement et de recherche français ou étrangers, des laboratoires publics ou privés. 


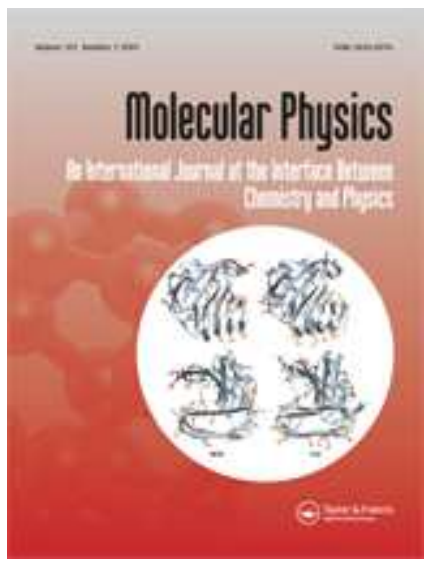

\section{Random phase approximation correlation energies with exact Kohn-Sham exchange}

\begin{tabular}{|c|c|}
\hline Journal: & Molecular Physics \\
\hline Manuscript ID: & TMPH-2009-0304 \\
\hline Manuscript Type: & Special Issue Paper - In honour of Prof Werner 60th birthday \\
\hline $\begin{array}{r}\text { Date Submitted by the } \\
\text { Author: }\end{array}$ & 30-Sep-2009 \\
\hline Complete List of Authors: & $\begin{array}{l}\text { Hesselmann, Andreas; Universitaet Erlangen, Lehrstuhl fuer } \\
\text { Theoretische Chemie } \\
\text { Goerling, Andreas; Universitaet Erlangen, Lehrstuhl fuer } \\
\text { Theoretische Chemie }\end{array}$ \\
\hline Keywords: & $\begin{array}{l}\text { random phase approximation, exact exchange, Kohn-Sham, orbital- } \\
\text { dependent functional, exact exchange kernel }\end{array}$ \\
\hline \multicolumn{2}{|c|}{$\begin{array}{l}\text { Note: The following files were submitted by the author for peer review, but cannot be converted } \\
\text { to PDF. You must view these files (e.g. movies) online. }\end{array}$} \\
\hline New WinZip File.zip & \\
\hline
\end{tabular}

\section{S ScholaroNE Manuscript Central}




\title{
RESEARCH ARTICLE
}

\section{Random phase approximation correlation energies with exact Kohn-Sham exchange}

\author{
Andreas Heßelmann and Andreas Görling \\ (Received 00 Month 200x; final version received 00 Month 200x)
}

Lehrstuhl für Theoretische Chemie, Universität Erlangen-Nürnberg, Egerlandstr. 3, D-91058 Erlangen, Germany

\begin{abstract}
The random phase approximation (RPA) correlation energy is expressed in terms of the exact local Kohn-Sham (KS) exchange potential and corresponding adiabatic and nonadiabatic exchange kernels for density-functional reference determinants. The approach naturally extends the RPA method in which, conventionally, only Coulomb interactions are included. By comparison with the coupled cluster singles doubles with perturbative triples method it is shown for a set of small molecules that the new RPA method based on KS exchange yields correlation energies more accurate than RPA on the basis of Hartree-Fock exchange.
\end{abstract}

\section{Introduction}

The description of electron correlation effects has been shown to be essential for an accurate description of molecular energetics, structures and properties. Because of this, in recent years, there has been much effort to improve the methods that are in use to determine correlated wave functions and energies and also the underlying algorithms that are used for their computer implementation. Concering the latter point the most significant developments probably were density-fitting methods $[1-5]$ that reduce the computational cost with respect to the basis set size and secondly methods that treat electron correlation using localised orbitals [5-9].

The nowadays most widely used approaches to describe electron correlation effects are Møller-Plesset (MP) perturbation theory [10-14], coupled-cluster (CC) methods $[11,14,15]$ and density-functional theory (DFT) [14, 16-18]. Principally all three approaches aim at describing electron correlation exactly, i.e. they offer the possibility to obtain the exact solutions of the nonrelativistic Schrödinger equation. In praxis, however, approximations have to be introduced: in MP and CC theory the wave function is usually restricted to include at most triple excitations from the given reference determinant while in the case of DFT the yet unknown exchange-correlation (xc) functional has to be approximated.

The most prominent approximations of the xc functional are the local density approximation (LDA) [16-18] and generalised gradient approximation (GGA) functionals [16-20] that describe the xc energy in terms of the electron density and its gradient. More recently these methods were further improved by introducing functionals depending on the laplacian of the density or the kinetic energy density (meta GGA's) [21-24] or that include fractions of nonlocal exchange (hyper GGA's) [25-28]. Unfortunately it was found that GGA's and many of their extensions suffer from a number of shortcomings, most importantly they are unable to describe long-range correlation effects or dispersion interactions [18, 29].

An important step forward in the development of density functionals was there-

ISSN: 0040-5167 print/ISSN 1754-2278 online

(C) $200 \mathrm{x}$ Taylor \& Francis

DOI: $10.1080 / 0040516 \mathrm{YYxxxxxxxx}$

http://www.informaworld.com 
fore to introduce functionals that are dependent both on occupied and unoccupied molecular orbitals and thus only implicitly dependent on the electron density [3047]. While such orbital-dependent functionals on the one hand have the advantage that exchange interactions can be treated exactly [35-37, 41, 48, 49] and thus the self-interaction error of the Coulomb interaction term is cancelled exactly, on the other hand, within the framework of orbital-dependent functionals, accurate expressions for the correlation functional can be obtained from many-body perturbation theory [31, 33, 34, 45, 50-55] or coupled-cluster theory [42]. Indeed it has been demonstrated that orbital-dependent functionals derived from second-order perturbation theory are capable to describe dispersion interactions [56] and yield improved total energies and molecular properties compared with standard GGA functionals $[39,40]$. There are, however, a number of points that limit the use of correlation functionals derived from perturbation theory: first the standard secondorder correlation energy expression is variationally unstable $[52,57]$ and so has to be modified in the energy denominators [40, 45], second the use of perturbation theory methods is problematic for extended and periodic systems which have a small or vanishing band gap. Finally it was shown in case of Møller-Plesset perturbation theory that the perturbation expansion of the correlation energy or of molecular properties often diverges [58-61].

Because of this there is the need to derive new orbital-dependent functionals that sum certain types of perturbation diagrams up to infinity as is for example done in coupled-cluster theory. One such method is the random phase approximation (RPA) $[12,13,62-70]$ which can be seen as an approximate coupled-cluster doubles (CCD) approach in which the doubles amplitudes are restricted to those portions that lead to ring diagrams $[12,71]$.

The name random phase approximation stems from a classical mechanical treatment of the collective properties of the electron gas by Bohm and Pines [72, 73]. Later on Nozieres and Pines demonstrated that in a quantum-mechanical framework RPA is equivalent to the addition of singly excited determinants to an independent particle determinant [74] and Ehrenreich et al. have then shown the equivalence with the perturbed self-consistent field method [75]. Historically, however, the earliest RPA calculations were not based on antisymmetrised wave functions and RPA was originally associated with ring sums for the electron gas in which the interactions were not antisymmetrised. This term of the RPA is still used and usually refered to in solid state physics (see e.g. Ref. [76]) while in quantum chemistry the term RPA is often used as another term for time-dependent Hartree-Fock (TDHF) because RPA here is used mainly for the determination of excitation energies and response properties $[13,77-79]$. In order to avoid the confusion that arises due to these different definitions it has become common to term RPA methods including exchange interactions either 'RPA with exchange' or 'full RPA' while RPA methods excluding exchange terms are termed just RPA or 'direct RPA'.

The RPA method without exchange has been shown to yield the exact correlation energy of the electron gas in the high density limit $[12,63]$ and thus may be very useful for larger and particularly extended electronic systems. However, from a diagrammatical point of view 'direct RPA' has the disadvantage that it contains many exclusion principle violating (EPV) diagrams whose cancelling counterparts are absent. This deficiency is most severe for small systems with small basis sets and this might explain that it did not play a significant role in quantum chemical treatments.

This is different for the 'RPA with exchange' which apart from its use for the calculation of response properties and excitation energies has also been used to directly determine non-multipole expanded dispersion energies [13,80-82] and total 


\section{September 30,2009
Page 3 of 22

correlation energies [83-86]. In 1977 Szabo and Ostlund analysed the full RPA correlation contribution to the interaction energy of two remote molecules and showed that the dispersive part of the interaction is described on the coupled HartreeFock level (identical with TDHF) [84]. The 'full RPA' method should therefore be more suited to describe intermolecular interactions than second-order MøllerPlesset theory which describes the dispersion energy contribution only on an uncoupled Hartree-Fock level [84, 87]. However, the full RPA method nevertheless has not been widely used for the determination of correlation energies and this may stem from the fact that coupled-cluster methods turned out to be more accurate while not much more expensive.

Very recently a revival of RPA methods in quantum chemistry has occured as RPA was found to be an attractive starting point for the development of new orbitaldependent density functionals [44, 46, 71, 88-94]. Compared with the second-order perturbation theory functionals described above, RPA based functionals do not possess a variational instability, can be applied also to extended systems with small band gaps and they describe dispersion interactions on a coupled TDDFT level which has been found to give very accurate dispersion energies in the framework of the DFT-SAPT (symmetry-adapted intermolecular perturbation theory) method [95]. Moreover, Furche has shown [90] that the correlation energy of the direct RPA method can be computed with an efficient algorithm that scales only as $\mathcal{N}^{5}$ with the molecular size and therefore the computational cost does not exceed the cost from perturbation theory based functionals. However, while for bulk properties of solids the direct RPA method has been shown to give a good agreement with experimental results [76], for molecular systems its performance for, e.g., the prediction of atomization energies was found to be not much better than with standard GGA functionals [89]. Because of this the exchange(-correlation) interactions omitted in direct RPA have to be accounted for and a straightforward approach to do so is the inclusion of local exchange-correlation kernels derived from the functional derivatives of GGA functionals [44]. It has been shown that in conjunction with hybrid functionals this extension to the RPA, termed fluctuation-dissipation theorem DFT (FDT-DFT), yields atomization energies and intermolecular interactions that outperform direct RPA results [44]. However, it has been shown that the use of local xc kernels in the framework of FDT-DFT leads to unphysical pair density functions that diverge for small interelectronic distances and this apparently leads to a slow basis set convergence of correlation energies [44].

One solution to this problem consists in a separation of the electron interaction into a short-range and a long-range part and to treat the RPA correlation for the long-range part only while the short-range interactions are described using GGA functionals. Such short-range-long-range functionals have very recently been developed by Scuseria et al. [92, 93] and Toulouse et al. [94]. Both types of functionals were especially tested for the description of van-der-Waals interactions and it was found that they perform exceedingly better than direct RPA [93, 94]. It should be noted here that the long-range RPA part is different in both functionals: the functional from Scuseria et al. uses direct RPA only while the functional from Toulouse et al. in addition contains the exchange kernel according to the TDHF formalism. Another extension to the RPA correlation functional was proposed by Jiang and Engel that was based on adding the second-order exchange energy perturbation expression to the direct RPA energy [46]. This approach, however, leads to the same problematic properties of the functional as described above for the pure second-order perturbation theory functionals.

A natural extension to direct RPA functionals are full RPA functionals that account for exchange interactions by using directly the exact exchange kernel of DFT 
[96-98]. The exact exchange kernel, that is the functional derivative of the exact KS-exchange potential with respect to the electron density in contrast to standard GGA kernels is both nonlocal and frequency-dependent and therefore does not lead to an unphysical behaviour of the pair density for small interelectronic distances. The exact KS exchange potential is determined and used in EXX (exactexchange) methods [35, 36, 41] within the framework of the optimised effective potential method $[31,99,100]$ that yields derivatives of orbital-dependent functionals with respect to the electron density. First implementations of the adiabatic and nonadiabatic KS exchange kernel [101, 102], however, relying on expansions of the Kohn-Sham response function in auxiliary basis sets suffered from numerical instabilities and are therefore impractical for the accurate determination of response properties and energies. We have recently implemented the exact-exchange kernel using a modified TDDFT method (Ref. [103], see also section 2.3) and this new approach has been used to calculate excitation energies of weakly bound dimers, demonstrating that the time-dependent EXX method is capable to describe chargetransfer excitations. Independently a time-dependent EXX method for atomic systems using cubic splines radial basis functions has been implemented by Hellgren and von Barth and it was shown that this approach yields excitation energies and dispersion coefficients that are close to the corresponding TDHF results [91, 104]. Furthermore Hellgren and von Barth have also calculated correlation energies using RPA with the exact-exchange kernel for a few closed-shell atomic systems and report a significantly good agreement with accurate configuration interaction $(\mathrm{CI})$ correlation energies [91].

In this work a RPA method with exact KS exchange (RPA(EXX)) is presented that is applicable to general molecular systems. For this the TDDFT formalism for orbital-dependent xc kernels from Ref. [103] will be used that avoids the numerically problematic inversion of the Kohn-Sham response matrices. It will be shown in section 2.3 that from the full RPA(EXX) method two further approximations can be devised, namely an RPA method including only the adiabatic exact-exchange kernel $(\operatorname{RPA}(\mathrm{AEXX}))$ and an RPA method with an exchange kernel obtained by scaling the nonadiabatic part of the full exact-exchange kernel with one half (RPA(EXXh)). Section 4 shows and discusses the results for total energies, correlation energies and reaction energies for a set of small organic molecules. Finally section 5 summarises and concludes.

\section{Theory}

\subsection{Electron correlation energies from the fluctuation-dissipation theorem}

The electronic energy of a many-body interacting system is usually split into the reference energy of a single-determinant wave function and a remainder termed the correlation energy. The reference energy is given by:

$$
E_{0}=\left\langle\Phi_{0}|\hat{H}| \Phi_{0}\right\rangle=\sum_{i}^{\text {occ }} 2\left\langle\phi_{i}\left|-\frac{1}{2} \nabla^{2}+v_{\text {ext }}\right| \phi_{i}\right\rangle+\sum_{i j}^{\text {occ }}[2(i i \mid j j)-(i j \mid i j)]
$$

where $\Phi_{0}$ is the reference determinant, $\hat{H}$ is the electronic hamilton operator, $\phi_{i}$ denotes an occupied orbital, $v_{\text {ext }}$ is the external potential (usually containing electronnucleus interactions) and $(i j \mid k l)$ is a two-electron integral in chemists notation. Note that, in the following, for convenience closed-shell formalism will be considered and spin will be taken into account by appropriate prefactors. Occupied orbitals shall 
be labelled by $i, j, k, \ldots$ and unoccupied orbitals by $a, b, c, \ldots$. The definition of the correlation energy:

$$
E_{c}=\langle\Psi|\hat{H}| \Psi\rangle-\left\langle\Phi_{0}|\hat{H}| \Phi_{0}\right\rangle
$$

depends on the reference determinant $\Phi_{0}$ which a priory is not specified ( $\Psi$ may be the exact or an approximation to the exact wave function). If $\Phi_{0}$ is chosen as the HF determinant the definition of the correlation energy results that is usually refered to in wave-function based quantum chemistry methods. In density-functional theory $\Phi_{0}$ is the KS determinant. This leads to another definition of the correlation energy. In this work the reference determinant will be the EXX determinant with orbitals obtained from an exact-exchange method [41] which does not yield Hartree-Fock but EXX orbitals.

Using the fluctuation-dissipation theorem $[105,106]$ the correlation energy of the interacting many-body system can be obtained from a coupling strength integration over the Coulomb-type integral of the correlated part of the pair correlation function $P_{\alpha}^{c}[44,105,106]$ :

$$
\begin{aligned}
E_{c} & =\frac{1}{2} \int_{0}^{1} d \alpha \int d \mathbf{r}_{1} d \mathbf{r}_{2} \frac{P_{\alpha}^{c}\left(\mathbf{r}_{1}, \mathbf{r}_{2}\right)}{r_{12}} \\
& =-\frac{1}{2 \pi} \int_{0}^{1} d \alpha \int d \mathbf{r}_{1} d \mathbf{r}_{2} \frac{1}{r_{12}} \int_{0}^{\infty} d \omega\left(\chi_{\alpha}\left(\mathbf{r}_{1}, \mathbf{r}_{2}, \omega\right)-\chi_{0}\left(\mathbf{r}_{1}, \mathbf{r}_{2}, \omega\right)\right)
\end{aligned}
$$

where $\alpha$ is the coupling strength, $\chi_{0}$ is the uncoupled response function and $\chi_{\alpha}$ is the coupled response function at coupling strength $\alpha$. The integration over $\omega$ goes over imaginary frequencies, i.e. here and in the following $\omega$ is defined to be an imaginary quantity. The uncoupled response function is given in terms of the orbitals and eigenvalues of the noninteracting KS system:

$$
\chi_{0}\left(\mathbf{r}_{1}, \mathbf{r}_{2}, \omega\right)=\sum_{i a} \frac{4 \varepsilon_{i a}}{\varepsilon_{i a}^{2}+\omega^{2}} \phi_{i a}\left(\mathbf{r}_{1}\right) \phi_{i a}\left(\mathbf{r}_{2}\right)
$$

where $\phi_{i a}(\mathbf{r})=\phi_{i}(\mathbf{r}) \phi_{a}(\mathbf{r})$ denotes an occupied-virtual orbital product and the convention $\varepsilon_{i a}=\varepsilon_{a}-\varepsilon_{i}$ is used. The coupled response function $\chi_{\alpha}$ can be obtained from the Dyson-type equation:

$\chi_{\alpha}\left(\mathbf{r}_{1}, \mathbf{r}_{2}, \omega\right)=\chi_{0}\left(\mathbf{r}_{1}, \mathbf{r}_{2}, \omega\right)+\int d \mathbf{r}_{3} d \mathbf{r}_{4} \chi_{0}\left(\mathbf{r}_{1}, \mathbf{r}_{3}, \omega\right)\left[\frac{\alpha}{r_{34}}+f_{x c}^{\alpha}\left(\mathbf{r}_{3}, \mathbf{r}_{4}, \omega\right)\right] \chi_{\alpha}\left(\mathbf{r}_{4}, \mathbf{r}_{2}, \omega\right)$

which is rewritten symbolically by

$$
\chi_{\alpha}=\chi_{0}+\chi_{0} W \chi_{\alpha}
$$

where $W=1 / r_{12}+f_{x c}$ is the interaction operator comprising Coulomb-, exchangeand correlation-effects. It can be seen that Eq.(4) can be solved iteratively obtaining an $n$-order expansion of the coupled response function:

$$
\chi_{\alpha}^{(n)}=\chi_{0}+\chi_{0} W \chi_{0}+\chi_{0} W \chi_{0} W \chi_{0}+\ldots
$$


and in infinite order the interacting response function is given by:

$$
\begin{aligned}
\chi_{\alpha} & =\left(1-\chi_{0} W\right)^{-1} \chi_{0} \\
& =\left(\chi_{0}^{-1}-W\right)^{-1}
\end{aligned}
$$

The response function can also be written as (we assume in the following an implict dependency on the coupling strength $\alpha$ )

$$
\chi_{\alpha}\left(\mathbf{r}_{1}, \mathbf{r}_{2}, \omega\right)=\sum_{p} \sum_{i a, j b} \frac{4 \omega_{p}}{\omega_{p}^{2}+\omega^{2}} U_{p, i a} U_{p, j b} \phi_{i a}\left(\mathbf{r}_{1}\right) \phi_{j b}\left(\mathbf{r}_{2}\right)
$$

where $\omega_{p}$ are the excitation energies and $\mathbf{U}_{p}$ are the corresponding eigenvectors of the TDDFT eigenvalue equation:

$$
\left[\varepsilon^{2}-4 \varepsilon^{1 / 2} \mathbf{K}(\omega) \varepsilon^{1 / 2}\right] \mathbf{U}=\omega^{2} \mathbf{U}
$$

with

$$
K_{i a, j b}(\omega)=\int d \mathbf{r}_{1} d \mathbf{r}_{2} \phi_{i a}\left(\mathbf{r}_{1}\right) W\left(\mathbf{r}_{1}, \mathbf{r}_{2}, \omega\right) \phi_{j b}\left(\mathbf{r}_{2}\right)
$$

and $\varepsilon$ is a diagonal matrix with the elements $\varepsilon_{i a, j b}=\delta_{i a, j b}\left(\varepsilon_{a}-\varepsilon_{i}\right)$.

It is now assumed that the coupling matrix $\mathbf{K}$ and thus the eigenvectors $\mathbf{U}$ are frequency-independent. Then the $\omega$-integration in Eq.(2) can be done analytically. If in addition also the Coulomb-type integrals over $\mathbf{r}_{1}$ and $\mathbf{r}_{2}$ are performed the correlation energy can be rewritten as:

$$
\begin{aligned}
E_{c} & =-\int_{0}^{1} d \alpha\left[\sum_{p} \sum_{i a, j b} U_{p, i a} U_{p, j b}(i a \mid j b)-\sum_{i a}(i a \mid i a)\right] \\
& =-\int_{0}^{1} d \alpha\left[\sum_{p} \sum_{i a, j b} U_{p, i a} U_{p, j b}(i a \mid j b)\right]+\frac{1}{2} \sum_{i a}(i a \mid i a)
\end{aligned}
$$

If however the xc-kernel is frequency dependent the correlation energy would have to be obtained from:

$$
E_{c}=-\frac{1}{2 \pi} \int_{0}^{1} d \alpha \int_{0}^{\infty} d \omega\left[\sum_{p} \sum_{i a, j b} \frac{4 \omega_{p}}{\omega_{p}^{2}+\omega^{2}} U_{p, i a}(\omega) U_{p, j b}(\omega)(i a \mid j b)\right]+\frac{1}{2} \sum_{i a}(i a \mid i a)
$$

Alternatively the computation of the correlation energy can also be done without solving the eigenvalue equation (7). For this we define:

$$
\begin{aligned}
\lambda_{i a, j b}(\omega) & =\delta_{i a, j b} \frac{4 \varepsilon_{a i}}{\varepsilon_{a i}^{2}+\omega^{2}} \\
K_{i a, j b}(\omega) & =\left(i a\left|\frac{1}{r_{12}}+f_{x c}(\omega)\right| j b\right) \\
\boldsymbol{\chi}_{\alpha} & =(\mathbf{1}-\alpha \boldsymbol{\lambda} \mathbf{K})^{-1} \boldsymbol{\lambda}
\end{aligned}
$$


(compare with Eq.(6)) so that $E_{c}$ can be written as:

$$
E_{c}=-\frac{1}{2 \pi} \int_{0}^{1} d \alpha \int_{0}^{\infty} d \omega \sum_{i a, j b}\left(\left(\chi_{\alpha}\right)_{i a, j b}-\lambda_{i a, j b}\right)(i a \mid j b)
$$

Note that of course again the uncoupled part involving an $\alpha$ - and $\omega$-integration of the $\lambda$-term can be separated as in Eq.(8) and again yields the term $\frac{1}{2} \sum_{i a}(i a \mid i a)$.

\subsection{Coupling strength integration}

If the exchange-correlation kernel $f_{x c}^{\alpha}$ is approximated by the exchange-kernel $f_{x}^{\alpha}=$ $\alpha f_{x}^{1}=\alpha f_{x}$ with $f_{x}=f_{x}^{\alpha=1}$ then the electron interaction operator $W_{\alpha}=\frac{\alpha}{r_{12}}+f_{x c}^{\alpha}$ turns into $W_{\alpha}=\alpha W_{1}=\alpha W=\alpha\left(\frac{1}{r_{12}}+f_{x}\right)$ with $W=W_{\alpha=1}$ and depends linearly on the coupling strength. The response function at coupling strength $\alpha$ then is given by:

$$
\chi_{\alpha}=\chi_{0}+\alpha \chi_{0} W \chi_{0}+\alpha^{2} \chi_{0} W \chi_{0} W \chi_{0}+\ldots
$$

and in the occupied-virtual orbital product space (Eq. (9)):

$$
\chi_{\alpha}=\boldsymbol{\lambda}+\alpha \boldsymbol{\lambda} \mathbf{K} \boldsymbol{\lambda}+\alpha^{2} \boldsymbol{\lambda} \mathbf{K} \boldsymbol{\lambda} \mathbf{K} \boldsymbol{\lambda}+\ldots
$$

so that the coupling strength integration gives:

$$
\int_{0}^{1} d \alpha \boldsymbol{\chi}_{\alpha}=\boldsymbol{\lambda}+\frac{1}{2} \boldsymbol{\lambda K} \boldsymbol{\lambda}+\frac{1}{3} \boldsymbol{\lambda K} \boldsymbol{\lambda} \mathbf{K} \boldsymbol{\lambda}+\frac{1}{4} \boldsymbol{\lambda} \mathbf{K} \boldsymbol{\lambda} \mathbf{K} \boldsymbol{\lambda} \mathbf{K} \boldsymbol{\lambda}+\ldots
$$

It is now convenient to exploit the fact that $\boldsymbol{\lambda}$ is diagonal and its square root can easily be taken. Using $\tilde{\mathbf{K}}=\boldsymbol{\lambda}^{1 / 2} \mathbf{K} \boldsymbol{\lambda}^{1 / 2}$ the coupling strength integrated response matrix can be rewritten:

$$
\int_{0}^{1} d \alpha \boldsymbol{\chi}_{\alpha}=\boldsymbol{\lambda}^{1 / 2}\left[\mathbf{1}+\frac{1}{2} \tilde{\mathbf{K}}+\frac{1}{3} \tilde{\mathbf{K}} \tilde{\mathbf{K}}+\frac{1}{4} \tilde{\mathbf{K}} \tilde{\mathbf{K}} \tilde{\mathbf{K}}+\ldots\right] \boldsymbol{\lambda}^{1 / 2}
$$

Compared with the power series:

$$
-\ln (1-x)=x+\frac{1}{2} x^{2}+\frac{1}{3} x^{3}+\frac{1}{4} x^{4}+\ldots
$$

the coupling strength integrated response function can be expressed by

$$
\int_{0}^{1} d \alpha \boldsymbol{\chi}_{\alpha}=\boldsymbol{\lambda}^{1 / 2}\left[-\ln (1-\tilde{\mathbf{K}}) \tilde{\mathbf{K}}^{-1}\right] \boldsymbol{\lambda}^{1 / 2}
$$

The logarithm of the symmetric matrix $(1-\tilde{\mathbf{K}})$ can be evaluated analytically as:

$$
\ln (\mathbf{1}-\tilde{\mathbf{K}})=\mathbf{U}^{T} \ln (\boldsymbol{\Lambda}) \mathbf{U}
$$

where the matrix $\mathbf{U}$ contains the eigenvectors and $\boldsymbol{\Lambda}$ is a diagonal matrix containing the eigenvalues of the symmetric matrix $(\mathbf{1}-\tilde{\mathbf{K}})$. Note that the precondition for applying Eq. (13) is of course that the matrix $(\mathbf{1}-\tilde{\mathbf{K}})$ is positive definite. This was the case for all systems that were studied in this work and so the logarithm 


\subsection{FD-DFT using the exact-exchange kernel}

The frequency dependent exact-exchange kernel can generally be written as [96-98]:

$$
f_{x}\left(\mathbf{r}_{1}, \mathbf{r}_{2}, \omega\right)=\int d \mathbf{r}_{3} d \mathbf{r}_{4} \chi_{0}^{-1}\left(\mathbf{r}_{1}, \mathbf{r}_{3}, \omega\right) h_{x}\left(\mathbf{r}_{3}, \mathbf{r}_{4}, \omega\right) \chi_{0}^{-1}\left(\mathbf{r}_{4}, \mathbf{r}_{2}, \omega\right)
$$

with $h_{x}=h_{x}^{[1]}+h_{x}^{[2]}$ given by (note that $\omega$ is assumed to be imaginary, so for real frequencies the signs in front of the $\omega^{2}$ contributions would have to be changed):

$$
\begin{aligned}
h_{x}^{[1]}\left(\mathbf{r}_{1}, \mathbf{r}_{2}, \omega\right)= & \sum_{i j, a b}\left[\frac{-4 \varepsilon_{i a} \varepsilon_{j b}+4 \omega^{2}}{\left(\varepsilon_{i a}^{2}+\omega^{2}\right)\left(\varepsilon_{j b}^{2}+\omega^{2}\right)}\left((i j \mid a b)+\delta_{a b}\left\langle i\left|\hat{v}_{x}^{\mathrm{NL}}-\hat{v}_{x}\right| j\right\rangle-\delta_{i j}\left\langle a\left|\hat{v}_{x}^{\mathrm{NL}}-\hat{v}_{x}\right| b\right\rangle\right)+\right. \\
& \left.+\frac{-4 \varepsilon_{i a} \varepsilon_{j b}-4 \omega^{2}}{\left(\varepsilon_{i a}^{2}+\omega^{2}\right)\left(\varepsilon_{j b}^{2}+\omega^{2}\right)}(i b \mid j a)\right] \phi_{i a}\left(\mathbf{r}_{1}\right) \phi_{j b}\left(\mathbf{r}_{2}\right) \\
h_{x}^{[2]}\left(\mathbf{r}_{1}, \mathbf{r}_{2}, \omega\right)= & \sum_{i j, a} \frac{-4 \varepsilon_{j a}}{\varepsilon_{i a}\left(\varepsilon_{j a}^{2}+\omega^{2}\right)}\left\langle i\left|\hat{v}_{x}^{\mathrm{NL}}-\hat{v}_{x}\right| a\right\rangle\left[\phi_{i j}\left(\mathbf{r}_{1}\right) \phi_{j a}\left(\mathbf{r}_{2}\right)+\phi_{j a}\left(\mathbf{r}_{1}\right) \phi_{i j}\left(\mathbf{r}_{2}\right)\right]+ \\
& +\sum_{i, a b} \frac{-4 \varepsilon_{i b}}{\varepsilon_{i a}\left(\varepsilon_{i b}^{2}+\omega^{2}\right)}\left\langle i\left|\hat{v}_{x}^{\mathrm{NL}}-\hat{v}_{x}\right| a\right\rangle\left[\phi_{a b}\left(\mathbf{r}_{1}\right) \phi_{i b}\left(\mathbf{r}_{2}\right)+\phi_{i b}\left(\mathbf{r}_{1}\right) \phi_{a b}\left(\mathbf{r}_{2}\right)\right]
\end{aligned}
$$

with $\left\langle i\left|\hat{v}_{x}^{\mathrm{NL}}-\hat{v}_{x}\right| j\right\rangle$ giving the differences of the matrix elements of the nonlocal $\left(\hat{v}_{x}^{\mathrm{NL}}\right)$ and local $\left(\hat{v}_{x}\right)$ exchange potentials. Unfortunately the computation of the exact-exchange kernel via Eq. (14) using finite basis sets is problematic due to the occurence of the inverses of the uncoupled response functions $\chi_{0}$. Because of this in Ref. [103] the TDDFT response equations were reformulated yielding the response to the effective Kohn-Sham potential and not, as usually, the electron density. With this a close approximation of the exact-exchange kernel is obtained that does not involve the inverses of the KS response functions anymore and that would be the exact functional derivative of the exact local KS exchange potential if the occupiedvirtual orbital products were linearly independent. As in case of the exact-exchange kernel defined in Eqs. (14-16) it can be split into two parts and the first part can be written as [103]:

$$
\begin{aligned}
{\left[K_{x}^{[1]}\right]_{i a, j b}=} & {\left[1-\frac{\omega^{2}}{\varepsilon_{i a} \varepsilon_{j b}}\right]\left[-(i j \mid a b)+\delta_{i j}\left\langle a\left|\hat{v}_{x}^{\mathrm{NL}}-\hat{v}_{x}\right| b\right\rangle-\delta_{a b}\left\langle i\left|\hat{v}_{x}^{\mathrm{NL}}-\hat{v}_{x}\right| j\right\rangle\right] } \\
& -\left[1+\frac{\omega^{2}}{\varepsilon_{i a} \varepsilon_{j b}}\right](i b \mid j a)
\end{aligned}
$$

while the second part, corresponding to Eq. (16), contains the occupied-unoccupied matrix elements $\left\langle i\left|\hat{v}_{x}^{\mathrm{NL}}-\hat{v}_{x}\right| a\right\rangle$. While its computation is more demanding than the 
computation of $K_{x}^{[1]}$ (see Ref. [103] for details) it has been found that its inclusion has a negligible effect for excitation energies or correlation energies of small molecules. This is concordant with the fact that the EXX orbitals can approximately be obtained from an occupied-occupied virtual-virtual unitary transformation of the Hartree-Fock orbitals which is explicitly exploited in approximate exact-exchange density functional theories $[37,49]$. Therefore the $K_{x}^{[2]}$ contribution has not been included in the calculations of this work.

Since the exchange-kernel of Eq. (17) is frequency-dependent we can define two additional approximations that will be used in the calculations:

adiabatic approximation (AEXX): $\omega$-dependent terms in Eq. (17) are omitted

EXXh: $\omega$-dependent terms in Eq. (17) are scaled with one half

It will now be shown that the correlation energy defined in terms of the exchangekernel of Eq. (17) is related to the leading, i.e., second order, term in perturbation theory along the adiabatic connection which is the analogue of Møller-Plesset perturbation theory within the KS formalism [31, 100]. In order to show this the power series of Eq. (11) is truncated after the second term and inserted in Eq. (10) yielding:

$$
\begin{aligned}
E_{c}^{(2)} & =-\frac{1}{2 \pi} \int_{0}^{1} d \alpha \int_{0}^{\infty} d \omega \sum_{i a, j b}\left(\alpha(\boldsymbol{\lambda} \mathbf{K} \boldsymbol{\lambda})_{i a, j b}\right)(i a \mid j b) \\
& =-\frac{1}{4 \pi} \int_{0}^{\infty} d \omega \sum_{i a, j b}\left((\boldsymbol{\lambda} \mathbf{K} \boldsymbol{\lambda})_{i a, j b}\right)(i a \mid j b)
\end{aligned}
$$

The coupling matrix $\mathbf{K}$ can be split into a Coulomb contribution and an exchange contribution:

$$
\mathbf{K}=\mathbf{K}_{C}+\mathbf{K}_{x}
$$

with $\mathbf{K}_{x}$ given by Eq. (17) and $\left[K_{C}\right]_{i a, j b}=(i a \mid j b)$. Accordingly the second-order correlation energy is split into a Coulomb- and an exchange-part. For the Coulombpart the frequency integration yields (see also Ref. [89]):

$$
E_{c, C}^{(2)}=\frac{-2(i a \mid j b)(i a \mid j b)}{\varepsilon_{i a}+\varepsilon_{j b}}
$$

which is the direct part of the second-order correlation energy. For the exchangepart one can make use of the integrals

$$
\begin{aligned}
& \int_{0}^{\infty} d \omega \frac{x y-\omega^{2}}{\left(x^{2}+\omega^{2}\right)\left(y^{2}+\omega^{2}\right)}=0 \forall x^{2}, y^{2}, x y>0 \\
& \int_{0}^{\infty} d \omega \frac{x y+\omega^{2}}{\left(x^{2}+\omega^{2}\right)\left(y^{2}+\omega^{2}\right)}=\frac{\pi}{x+y} \forall x^{2}, y^{2}, x y>0
\end{aligned}
$$

and obtains:

$$
E_{c, x}^{(2)}=\frac{(i a \mid j b)(i b \mid j a)}{\varepsilon_{i a}+\varepsilon_{j b}}
$$

which shows that the first part of the exchange-kernel of Eq. (17) vanishes upon $\omega$-integration and does not contribute to the second-order correlation energy. The 


\section{Computational Details}

Total energies and correlation energies were calculated with the approach described in section 2 for a set of 21 small organic molecules. The geometries used in this work were optimised at the MP2 (second-order Møller-Plesset perturbation theory) level with the aug-cc-pVTZ basis set [114] and are the same as those used in Ref. [115].

The exact-exchange Kohn-Sham (EXX) orbitals and eigenvalues were obtained by first performing numerically stable EXX calculations with the method from Ref. [41] for all 21 molecules in twos steps. In the first step the exact local KS exchange potential was obtained with the balanced uncontracted triple-zeta orbital- and auxiliary basis sets of Ref. [41]. In the second step EXX orbitals and eigenvalues were calculated by using the exact local exchange-potential represented in the auxiliary basis set obtained in the first step in a subsequent EXX calculation with the smaller contracted aug-cc-pVTZ orbital basis set [114]. In the EXX calculation of the second step only the Coulomb-potential was optimised self-consistently. Correlation energies using the approach described in section 2 were then calculated using the EXX orbitals and eigenvalues as input, i.e., the calculations were performed in a post Kohn-Sham (post selfconsistent-field) way.

While the coupling-strength integration required for the computation of the correlation energies (see e.g. Eq. (2)) could be done analytically using the approach described in section 2.2 , the $\omega$-integration over imaginary frequencies can not be done analytically for frequency-dependent exchange-correlation kernels. In this work we have used a 12-point Gauss-Legendre quadrature [116] for the frequency integration which was found to be accurate to approximately $10^{-5}$ hartree.

For comparison the molecular energies were also calculated using Hartree-Fock (HF), MP2, coupled cluster with singles and doubles (CCSD) and additionally also perturbative triples (CCSD $(\mathrm{T})$ ), the Becke-Lee-Yang-Parr 3-parameter hybrid density functional (B3LYP) [25, 26], and the random-phase approximation with exact Hartree-Fock exchange (RPA(HF)). The correlation energy for the latter approach can be obtained from Eq. (8) if the response eigenvectors $\mathbf{U}$ are given by the solutions of the time-dependent Hartree-Fock equations [13, 79].

All calculations were done using the aug-cc-pVTZbasis set [114] and all electrons were correlated. The method described in section 2 has been implemented in the development version of the Molpro quantum chemistry package [117]. 


\section{ptember 30, 2009

\section{Results}

The total molecular energies calculated for the set of 21 molecules are displayed in table 1. The last three lines of the table show the root-mean square errors, the mean absolute errors and the relative percentual deviations from the CCSD(T) reference values of the fifth column. It can be observed that while the MP2 method generally captures over 90 percent of the correlation effect, with only few exceptions the CCSD energies are closer to the $\operatorname{CCSD}(\mathrm{T})$ reference results than the MP2 ones, as expected. In contrast to this the B3LYP method almost always yields total energies that are about $0.1-0.2$ hartree larger in magnitude than the respective CCSD $(\mathrm{T})$ values. On the other hand the RPA method with Hartree-Fock exchange $(\mathrm{RPA}(\mathrm{HF}))$ tends to underestimate the $\mathrm{CCSD}(\mathrm{T})$ energies slightly. It is interesting to note here that while $\mathrm{RPA}(\mathrm{HF})$ is superior to MP2 from the theoretical point of view (it sums up certain types of diagrams to infinity including all second-order diagrams), on average it does not give more accurate total energies than the MP2 method as can be obeserved from table 1. For example the mean absolute errors from RPA(HF) are about twice as large as the MAE's of the MP2 method for the given set of molecules. In contrast to this the RPA method with KS exchange $(\mathrm{RPA}(\mathrm{EXX}))$ gives total energies that are almost always closer to $\mathrm{CCSD}(\mathrm{T})$ than the MP2 energies and actually the RPA(EXX) energies are very close to the CCSD energies, exhibiting similar RMS errors and MAE. It can also be seen that the RPA(EXX) energies are always larger than their RPA(HF) counterparts. This is likely due to the different eigenvalue spectrum of the HF and EXX method, more precisely the EXX method yields bound unoccupied orbitals and thus smaller energy gaps between the occupied and lowest unoccupied molecular orbitals. In contrast to the RPA(EXX) energies the RPA energies from the adiabatic exchange kernel (RPA(AEXX)) always overestimate the CCSD $(\mathrm{T})$ energies and this lead us to devise the RPA(EXXh) approach with an EXX kernel in which the frequency dependent part is scaled by one half. The total energies of this method are shown in the last column of table 1 . It can be observed that the correspondence with the $\mathrm{CCSD}(\mathrm{T})$ reference values is excellent, the mean absolute deviations being only about 5 millihartree for the studied systems.

The diagram in figure 1 shows the deviations of the correlation energies of the respective methods from the $\mathrm{CCSD}(\mathrm{T})$ reference correlation energies. Note that the correlation energy displayed in Fig. 1 in all cases is defined as the difference of the total energies and the Hartree-Fock energies of the second column of table 1 in order to consider comparable values. Fig. 1 also shows that RPA(EXX) correlation energies are close to CCSD ones while being always larger than their RPA(HF) counterparts, again likely due to the different eigenvalue spectrum of HF and EXX. The diagram in Fig. 1 also shows clearly that the correlation energies of the RPA(EXXh) method interpolate between the RPA(AEXX) and RPA(EXX) energies and as a consequence are very close to the $\operatorname{CCSD}(\mathrm{T})$ reference correlation energies. This is also summarised in the diagram in Fig. 2 which displays the absolute deviations from the $\operatorname{CCSD}(\mathrm{T})$ correlation energies for all methods averaged over the 21 molecules studied in this work. Fig. 2 shows that the RPA(AEXX) and RPA(EXX) method clearly improve the accuracy of the correlation energy if compared with the corresponding RPA $(\mathrm{HF})$ method, but they have about the same average deviation of 25 millihartree as obtained with the MP2 and CCSD methods. On the other hand the RPA(EXXh) correlation energies deviate from the CCSD(T) reference energies only by 5 millihartree on average.

In order to assess the presented RPA approach with KS exchange for the prediction of reaction energies we have considered the 14 chemical reactions listed 
in table 2. Note that compared to the prediction of total energies this is an even more difficult test for electron correlation methods as the energy differences are typically an order of magnitude smaller than total (correlation) energies and for the reactions studied here they lie in the range of 8 to 80 millihartree. The input energies used for the calculation of the reaction energies all were taken from table 1. Figure 3 shows the statistical error estimates for all methods averaged over the 14 reactions with $\operatorname{CCSD}(\mathrm{T})$ as reference. Interestingly the B3LYP method that yields large deviations from $\operatorname{CCSD}(\mathrm{T})$ total energies gives a comparable accuracy for the chemical reactions of table 2 as MP2. The RPA(EXXh) method on the other hand yields mean absolute errors for the reaction energies of about $1.7 \mathrm{kcal} / \mathrm{mol}$ which is about $0.7 \mathrm{kcal} / \mathrm{mol}$ larger than the deviations obtained with the CCSD method, despite the rather accurate total energies obtained with the RPA(EXXh) method. It can however be observed from Fig. 3 that both, the RPA(EXX) and RPA(EXXh) methods improve upon the accuracy of $\mathrm{RPA}(\mathrm{HF})$ for the chemical reactions while $\mathrm{RPA}(\mathrm{AEXX})$ and RPA(HF) itself give reaction enthalpies comparable to MP2.

While the comparison with $\operatorname{CCSD}(\mathrm{T})$ reaction energies is certainly the ultimate test for any lower level electron correlation method, it may be more equitable to use the CCSD method as the reference approach since both, MP2 and the RPA methods account only for double excitations and thus are rather approximations to the CCSD method. Therefore deviations to CCSD for the chemical reactions are displayed in Fig. 4. Here it can now be seen that the RPA(EXX) and RPA(EXXh) methods perform clearly better than MP2 and even than the related RPA(HF) method. For example the root mean square errors are only 0.9 and $1.7 \mathrm{kcal} / \mathrm{mol}$ while the MP2 method has a root mean square error of nearly $12 \mathrm{kcal} / \mathrm{mol}$ for the chemical reactions. As expected the RPA(AEXX) does not perform as well as the approaches with frequency dependent kernels, but anyhow it performs as well as RPA(HF) and also improves the MP2 method.

\section{Summary}

Random phase approximation methods for the correlation energy including exchange interations have been developed for exact-exchange Kohn-Sham reference determinants using the recently developed TDDFT formalism for orbital-dependent $\mathrm{xc}$ functionals that avoids the numerically difficult inversion of the Kohn-Sham response matrix. The exchange kernel derived from this approach, in contrast to standard GGA kernels, is both nonlocal and frequency dependent and therefore improves upon the unphysical behaviour of the pair density at small interelectronic distances occuring in the case of local GGA kernels. Besides RPA with the full exact-exchange kernel (RPA(EXX)) two approximate methods were derived, one in which the frequency dependent part is completely neglected (adiabatic approximation, $\operatorname{RPA}(\operatorname{AEXX}))$ and one in which the frequency dependent part is scaled with one half (RPA(EXXh)).

It has been shown for a set of 21 small organic molecules that the RPA(EXX) method underestimates the correlation energy compared to coupled cluster singles doubles with perturbative triples $(\operatorname{CCSD}(\mathrm{T}))$ correlation energies while the RPA(AEXX)) overestimates the CCSD (T) correlation energies to about the same amount. Accordingly the RPA(EXXh) method yields correlation energies that are close to the $\operatorname{CCSD}(\mathrm{T})$ reference energies with an average deviation of only 0.005 hartree for the systems that were studied. All three RPA approaches with exact KS exchange give correlation energies that are on average in a much better agreement with CCSD(T) correlation energies than the corresponding RPA method including Hartree-Fock exact-exchange (RPA(HF)) and the RPA(EXXh) method clearly 
outperforms second-oder Møller-Plesset (MP2) and CCSD in this respect.

However, it was found that the reaction energies for 14 organic reactions obtained by all three introduced RPA methods are only slightly better than corresponding MP2 and RPA(HF) reaction energies if compared to $\operatorname{CCSD}(\mathrm{T})$ reference energies and gave worse results than CCSD. However, if compared to CCSD as reference method for the reaction energies the RPA(EXX) and RPA(EXXh) method performed clearly better than the MP2 and RPA(HF) method for the reactions that were studied. This finding is significant from the point of view that the computational cost of full RPA(EXX) calculations should be not much higher than that of MP2 if density-fitting methods are exploited, as is shown in Ref. [113], since both methods then scale as $\mathcal{N}^{5}$ with respect to the molecular size. The presented RPA(EXX) method is therefore an orbital-dependent functional that can be applied to large molecules and may become an attractive method for quantum chemistry applications in the future.

\section{Acknowledgements}

The authors gratefully acknowledge the funding of the German Research Council (DFG), which, within the framework of its 'Excellence Initiative', supports the Cluster of Excellence 'Engineering of Advanced Materials' (www.eam.uni-erlangen.de) at the University of Erlangen-Nuremberg. 


\section{References}

[1]E. J. Baerends, D. E. Ellis, and P. Ros, Chem. Phys. 2, 41 (1973).

[2]E. J. Baerends and P. Ros, Chem. Phys. 2, 52 (1973).

[3]P. M. W. Gill, B. G. Johnson, J. A. Pople, and S. W. Taylor, J. Chem. Phys. 96, 7178 (1992).

[4]B. I. Dunlap, Phys. Chem. Chem. Phys. 2, 2113 (2000).

[5]H.-J. Werner, F. R. Manby, and P. J. Knowles, J. Chem. Phys. 118, 8149 (2003).

[6]P. Pulay, Chem. Phys. Lett. 100, 151 (1983).

[7]S. Saebø and P. Pulay, Theor. Chem. Acc. 69, 357 (1986).

[8] C. Hampel and H.-J. Werner, J. Chem. Phys. 104, 6286 (1996).

[9]M. Schütz, G. Hetzer, and H.-J. Werner, J. Chem. Phys. 111, 5691 (1999).

[10]A. Szabo and N. S. Ostlund, Modern Qantum Chemistry (Dover press, 1996).

[11]T. Helgaker, P. Jørgensen, and J. Olsen, Molecular Electronic-Structure Theory (Wiley \& Sons, 2000).

[12]F. E. Harris, H. J. Monkhorst, and D. L. Freeman, Algebraic and diagrammatic methods in manyfermion theory (Oxford university press, 1992).

[13]R. McWeeny, Methods of molecular quantum mechanics (Academic Press, 1996).

[14] L. Piela, Ideas of quantum chemistry (Elsevier, 2007).

[15]I. S. und R. J. Bartlett, Many-Body Methods in Chemistry and Physics: MBPT and Coupled-Cluster Theory (Cambridge University Press, 2009).

[16]R. G. Parr and W. Yang, Density-Functional Theory of Atoms and Molecules (Oxford University Press, Oxford, 1989).

[17]R. M. Dreizler and E. K. U. Gross, Density Functional Theory (Springer, Heidelberg, 1990).

[18]W. Koch and M. C. Holthausen, A Chemist's Guide to Density Functional Theory (Wiley-VCH, 2001).

[19]B. G. Johnson, P. M. W. Gill, and J. A. Pople, J. Chem. Phys. 98, 5612 (1993).

$[20] \mathrm{K}$. Burke, J. P. Perdew, and W. Yang, Electronic density functional theory: Recent progress and new directions. (Plenum Press, New York, 1998).

[21]S. K. Ghosh and R. Parr, Phys. Rev. A 34, 785 (1986).

$[22]$ T. V. Voorhis and G. E. Scuseria, J. Chem. Phys. 109, 400 (1998).

[23] A. D. Boese and N. C. Handy, J. Chem. Phys. 116, 9559 (2002).

[24]J. Tao, J. P. Perdew, V. N. Staroverov, and E. Scuseria, Phys. Rev. Lett. (2003).

[25] A. D. Becke, J. Chem. Phys. 98, 1372 (1993).

[26]A. D. Becke, J. Chem. Phys. 98, 5648 (1993).

[27]C. Adamo and V. Barone, J. Chem. Phys. 110, 6158 (1999).

[28]J. P. Perdew, V. N. Staroverov, J. Tao, and G. E. Scuseria, Phys. Rev. A 78, 052513 (2008).

[29]T. van Mourik and R. J. Gdanitz, J. Chem. Phys. 116, 9620 (2002).

[30]T. Grabo, T. Kreibich, S. Kurth, and E. K. U. Gross, Strong Coulomb Correlations in Electronic Structure: beyond the Local Density Approximatio (Gordon \& Breach, Tokyo, 1998).

[31]A. Görling and M. Levy, Phys. Rev. A 50, 196 (1994).

[32]E. Engel, S. Keller, A. F. Bonetti, H. Müller, and R. M. Dreizler, Phys. Rev. A 52, 2750 (1995).

[33]E. Engel, A. F. Bonetti, S. Keller, I. Andrejkovics, and R. M. Dreizler, Phys. Rev. A 58, 964 (1998).

[34]E. Engel and R. M. Dreizler, J. Comp. Chem. 20, 31 (1999).

[35]A. Görling, Phys. Rev. Lett. 83, 5459 (1999).

[36]S. Ivanov, S. Hirata, and R. J. Bartlett, Phys. Rev. Lett. 83, 5455 (1999).

[37]F. Della Sala and A. Görling, J. Chem. Phys. 115, 5718 (2001).

[38]S. Hirata, S. Ivanov, I. Grabowski, R. Bartlett, K. Burke, and J. D. Talman, J. Chem. Phys. 115, 1635 (2001)

[39]R. J. Bartlett, V. F. Lotrich, and I. V. Schweigert, J. Chem. Phys. 123, 062205 (2005).

[40]R. J. Bartlett, I. V. Schweigert, and V. F. Lotrich, J. Mol. Struct. (Theochem) 771, 1 (2006).

[41] A. Heßelmann, A. Götz, F. D. Sala, and A. Görling, J. Chem. Phys. 127, 054102 (2007).

[42] A. Heßelmann, J. Chem. Phys. 122, 244108 (2005).

[43]A. Heßelmann, Phys. Chem. Chem. Phys. 8, 563 (2006)

[44]F. Furche and T. Van Voorhis, J. Chem. Phys. 122, 164106 (2005).

[45]H. Jiang and E. Engel, J. Chem. Phys. 125, 184108 (2006).

[46]H. Jiang and E. Engel, J. Chem. Phys. 127, 184108 (2007).

[47]C. Kollmar and M. Filatov, J. Chem. Phys. 128, 064101 (2008).

[48]J. B. Krieger, Y. Li, and G. J. Iafrate, Phys. Rev. A 46, 5453 (1992).

[49] O. V. Gritsenko and E. J. Baerends, Phys. Rev. A 64, 042506 (2001).

[50]R. J. Bartlett, I. Grabowski, S. Hirata, and S. Ivanov, J. Chem. Phys. 122, 034104 (2005).

[51]I. Grabowski, S. Hirata, S. Ivanov, and R. J. Bartlett, J. Chem. Phys. 116, 4415 (2002).

[52]P. Mori-Sánchez, Q. Wu, and W. Yang, J. Chem. Phys. 123, 062204 (2005).

[53]H. Jiang and E. Engel, J. Chem. Phys. 123, 224102 (2005).

[54]M. .Grüning, A. Marini, and A. Rubio, J. Chem. Phys. 124, 154108 (2006).

[55]E. Fabiano and F. D. Sala, J. Chem. Phys. 126, 214102 (2007).

[56]V. Lotrich, R. J. Bartletta, and I. Grabowski, Chem. Phys. Lett. 405, 43 (2005).

[57]D. Rohr, O. Gritsenko, and E. J. Baerends, Chem. Phys. Lett. 432, 336 (2006).

[58]N. C. Handy, P. J. Knowles, and K. Somasundram, Theor. Chim. Acta 68, 87 (1985).

[59]A. Halkier, H. Larsen, J. Olsen, and P. Jørgensen, J. Chem. Phys. 110, 7127 (1999).

[60]H. Larsen, A. Halkier, J. Olsen, and P. Jørgensen, J. Chem. Phys. 112, 1107 (2000).

[61]M. L. Leininger, W. D. Allen, and H. F. S. III, J. Chem. Phys. 112, 9213 (2000).

[62]A. D. MacLachlan and M. A. Ball, Rev. Mod. Phys. 36, 844 (1964).

[63]R. D. Mattuck, A guide to Feynman diagrams in the many-body problem (Dover press, 1992).

[64]J. V. Ortiz, J. Chem. Phys. 101, 6743 (1994).

[65]J. W. Negele and H. Orland, Quantum many-particle systems (Perseus publishing, Cambridge Massachusetts, 1998). 
[66]A. L. Fetter and J. D. Walecka, Quantum theory of many-particle systems (Dover press, 2002).

[67]D. B. Cook, Handbook of Computational Quantum Chemistry (Dover Press, 2004).

[68]J. Linderberg and Y. Öhrn, Propagators in quantum chemistry (Wiley Interscience, 2004).

[69] G. Czycholl, Theoretische Festkörperphysik (Springer Verlag, Berlin Heidelberg, 2004).

[70]G. F. Giuliani and G. Vignale, Quantum Theory of the Electron Liquid (Cambridge university press, 2005).

[71]G. E. Scuseria, T. M. Henderson, and D. C. Sorensen, J. Chem. Phys. 129, 231101 (2008).

[72]D. Bohm and D. Pines, Phys. Rev. 82, 625 (1951).

[73]D. Bohm and D. Pines, Phys. Rev. 85, 338 (1952).

[74]P. Nozieres and D. Pines, Nuovo Cimento [X]9, 470 (1958).

[75] H. Ehrenreich and M. H. Cohen, Phys. Rev. 115, 786 (1959).

[76]J. Harl and G. Kresse, Phys. Rev. Lett. 103, 056401 (1009).

[77]P. Jørgensen, Anu. Rev. Phys. Chem. 26, 359 (1975).

[78]G. Csanak, J. Phys. B: Atom. Molec. Phys. 7, 1289 (1974).

[79]J. Oddershede, P. Jørgensen, and D. L. Yeager, Comp. Phys. Rep. 2, 33 (1984).

[80]M. Jaszunski and R. McWeeny, Mol. Phys. 55, 1275 (1985).

[81]P. J. Knowles and W. J. Meath, Mol. Phys. 59, 965 (1986).

[82]P. J. Knowles and W. J. Meath, Chem. Phys. Lett. 124, 164 (1986).

[83]A. Szabo and N. S. Ostlund, Int. J. Quant. Chem. S11, 389 (1977).

[84] A. Szabo and N. S. Ostlund, J. Chem. Phys. 67, 4351 (1977).

[85]N. Ostlund and M. Karplus, Chem. Phys. Lett. 11, 450 (1971).

[86]J. Oddershede, Adv. Quant. Chem. 11, 275 (1978).

[87]S. M. Cybulski, G. Chalasinski, and R. Moszynski, J. Chem. Phys. 92, 4357 (1990).

[88]T. Kotani, J. Phys.: Condens. Matter 10, 9241 (1998).

[89]F. Furche, Phys. Rev. A 64, 195120 (2001).

[90]F. Furche, J. Chem. Phys. 129, 114105 (2008).

[91]M. Hellgren and U. von Barth, Phys. Rev. B 78, 115107 (2008).

[92]B. G. Janesko, T. M. Henderson, and G. E. Scuseria, J. Chem. Phys. 131, 034110 (2009).

[93]B. G. Janesko, T. M. Henderson, and G. E. Scuseria, J. Chem. Phys. 130, 081105 (2009).

[94]J. Toulouse, I. C. Gerber, G. Jansen, A. Savin, and J. Angyan, Phys. Rev. Lett. 102, 096404 (2009).

[95]A. Heßelmann and G. Jansen, Chem. Phys. Lett. 367, 778 (2003).

[96]A. Görling, Int. J. Quantum Chem. 69, 265 (1998).

[97]A. Görling, Phys. Rev. A 57, 3433 (1998).

[98]Y.-H. Kim and A. Görling, Phys. Rev. B 66, 035144 (2002)

[99]J. D. Talman and W. F. Shadwick, Phys. Rev. A 14, 36 (1976).

[100]A. Görling and M. Levy, Int. J. Quantum Chem. Symp. 29, 93 (1995).

[101]S. Hirata, S. Ivanov, I. Grabowski, and R. J. Bartlett, J. Chem. Phys. 116, 6468 (2002).

[102]Y. Shigeta, K. Hirao, and S. Hirata, Phys. Rev. A 73, 010502(R) (2006).

[103] A. Heßelmann, A. Ipatov, and A. Görling, Phys. Rev. A 80, 012507 (2009).

[104]M. Hellgrean and U. von Barth, J. Chem. Phys. 131, 044110 (2009).

[105]D. C. Langreth and J. P. Perdew, Solid State Commun. 17, 1425 (1975).

[106]Y. M. Niquet, M. Fuchs, and X. Gonze, Phys. Rev. A 68, 032507 (2003).

[107]N. J. Higham, Siam J. Matrix Anal. Appl. 22, 1126 (2001).

[108]J. R. Cardoso, S. Afr. Optom. (2005).

[109]N. Sherif and E. Morsy, Int. J. Algebra 2, 131 (2008).

[110]A. Görling, A. Heßelmann, M. Jones, and M. Levy, J. Chem. Phys. 128, 104104 (2008).

111]A. Heßelmann and A. Görling, Chem. Phys. Lett. 455, 110 (2008).

[112]R. Bukowski, R. Podeszwa, and K. Szalewicz, Chem. Phys. Lett. 414, 111 (2005).

[113]A. Heßelmann and A. Görling (????), to be published.

1114]R. A. Kendall, J. T. H. Dunning, and R. J. Harrison, J. Chem. Phys. 96, 6796 (1992).

[115]H.-J. Werner, T. B. Adler, and F. R. Manby, J. Chem. Phys. 126, 164102 (2007).

[116]R. D. Amos, N. C. Handy, P. J. Knowles, J. E. Rice, and A. J. Stone, J. Phys. Chem. 89, 2186 (1985).

[117]H.-J. Werner, P. J. Knowles, R. Lindh, M. Schütz, P. Celani, T. Korona, F. R. Manby, G. Rauhut, R. D. Amos, A. Bernhardsson, et al., MOLPRO, version 2008.2, a package of ab initio programs (2008), see http://www.molpro.net. 
Tables 


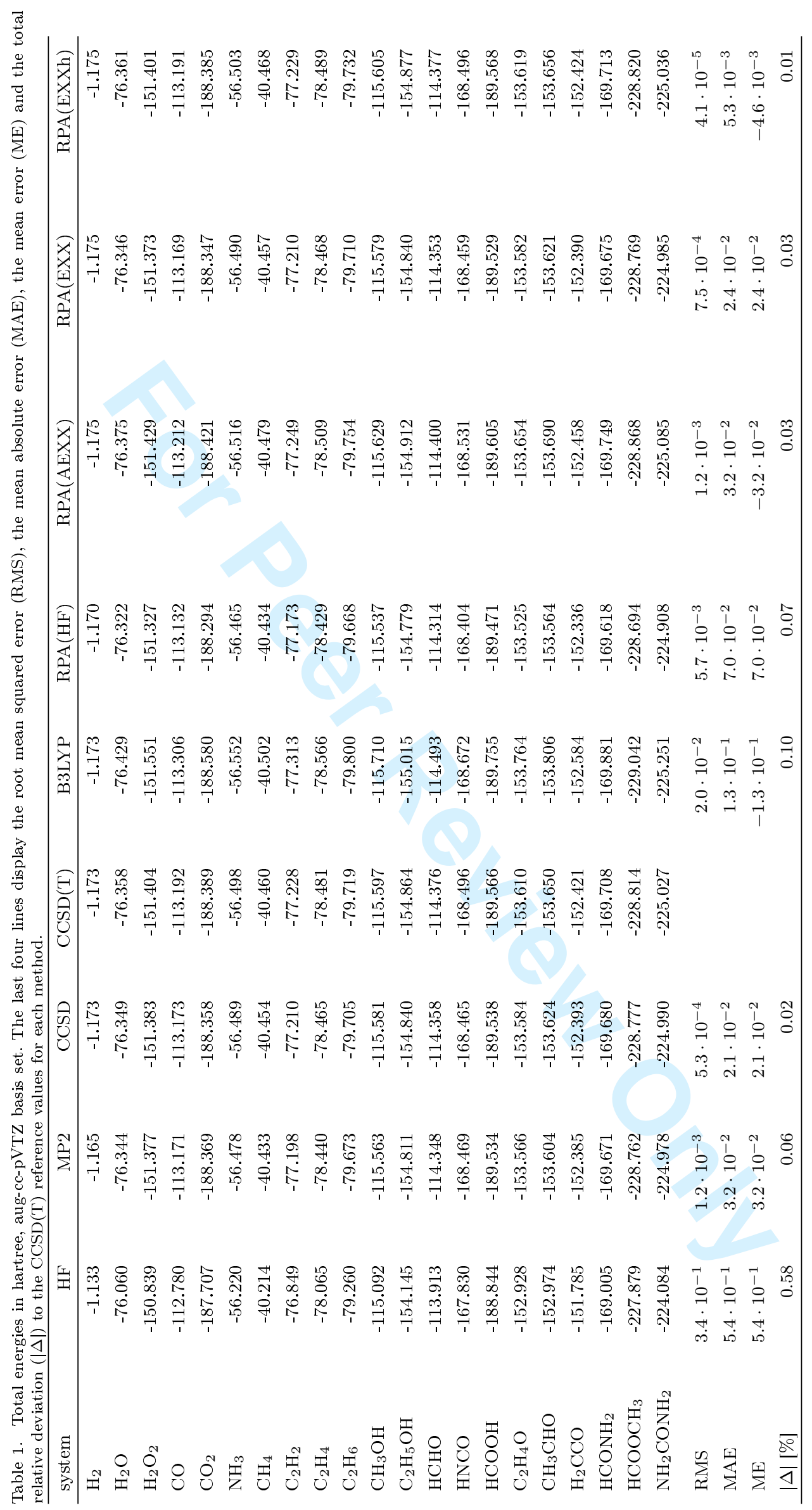


Table 2. Reactions

$\mathrm{C}_{2} \mathrm{H}_{2}+\mathrm{H}_{2} \quad \rightarrow \quad \mathrm{C}_{2} \mathrm{H}_{4}$

$\mathrm{C}_{2} \mathrm{H}_{4}+\mathrm{H}_{2} \quad \rightarrow \mathrm{C}_{2} \mathrm{H}_{6}$

$\mathrm{C}_{2} \mathrm{H}_{6}+\mathrm{H}_{2} \rightarrow 2 \mathrm{CH}_{4}$

$\mathrm{CO}+\mathrm{H}_{2} \rightarrow \mathrm{H}_{2} \mathrm{CO}$

$\mathrm{H}_{2} \mathrm{CO}+\mathrm{H}_{2} \rightarrow \mathrm{CH}_{3} \mathrm{OH}$

$\mathrm{H}_{2} \mathrm{O}_{2}+\mathrm{H}_{2} \rightarrow 2 \mathrm{H}_{2} \mathrm{O}$

$\mathrm{C}_{2} \mathrm{H}_{2}+\mathrm{H}_{2} \mathrm{O} \rightarrow \mathrm{CH}_{3} \mathrm{CHO}$

$\mathrm{C}_{2} \mathrm{H}_{4}+\mathrm{H}_{2} \mathrm{O} \rightarrow \mathrm{C}_{2} \mathrm{H}_{5} \mathrm{OH}$

$\mathrm{CH}_{3} \mathrm{CHO}+\mathrm{H}_{2} \rightarrow \mathrm{C}_{2} \mathrm{H}_{5} \mathrm{OH}$

$\mathrm{CO}+\mathrm{NH}_{3} \rightarrow \mathrm{HCONH}_{2}$

$\mathrm{CO}+\mathrm{H}_{2} \mathrm{O} \rightarrow \mathrm{CO}_{2}+\mathrm{H}_{2}$

$\mathrm{HNCO}+\mathrm{NH}_{3} \rightarrow \mathrm{NH}_{2} \mathrm{CONH}_{2}$

$\mathrm{CH}_{3} \mathrm{OH}+\mathrm{CO} \rightarrow \mathrm{HCOOCH}_{3}$

$\mathrm{CO}+\mathrm{H}_{2} \mathrm{O}_{2} \rightarrow \mathrm{CO}_{2}+\mathrm{H}_{2} \mathrm{O}$ 
Figures

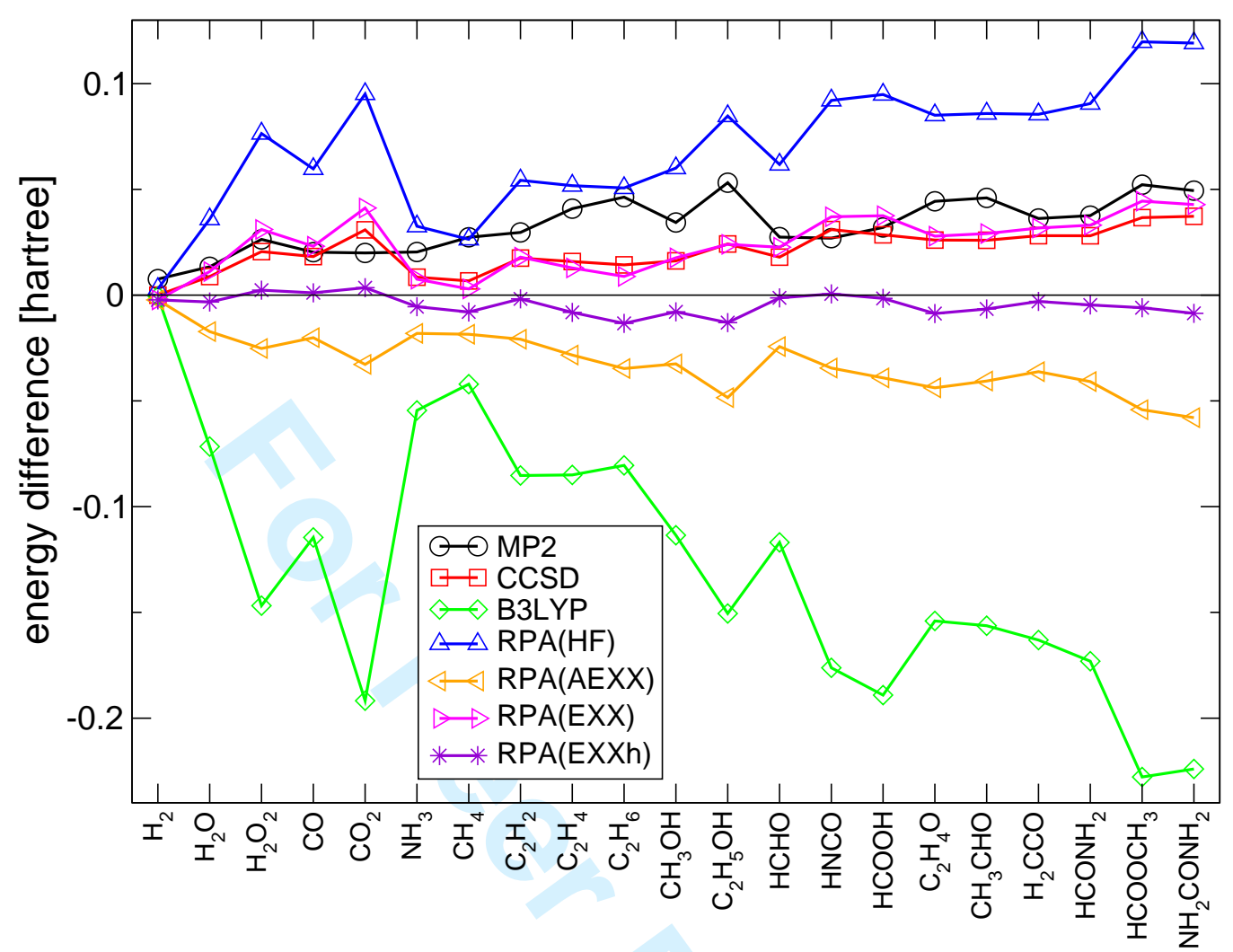

Figure 1. Deviations of correlation energies (defined in all cases as difference of the total energy with the HF total energy) from CCSD(T) correlation energies, aug-cc-pVTZ basis set.

2

3

6

7

9

10

11

13

14

15

17

18

19

21

22

23

24

26

27

28

30

31

32

33

34

35

36

37

38

39

40

41

42

43

44

45

46

47

48

49

50

51

52

53

54

55

56

57

58

59

60 


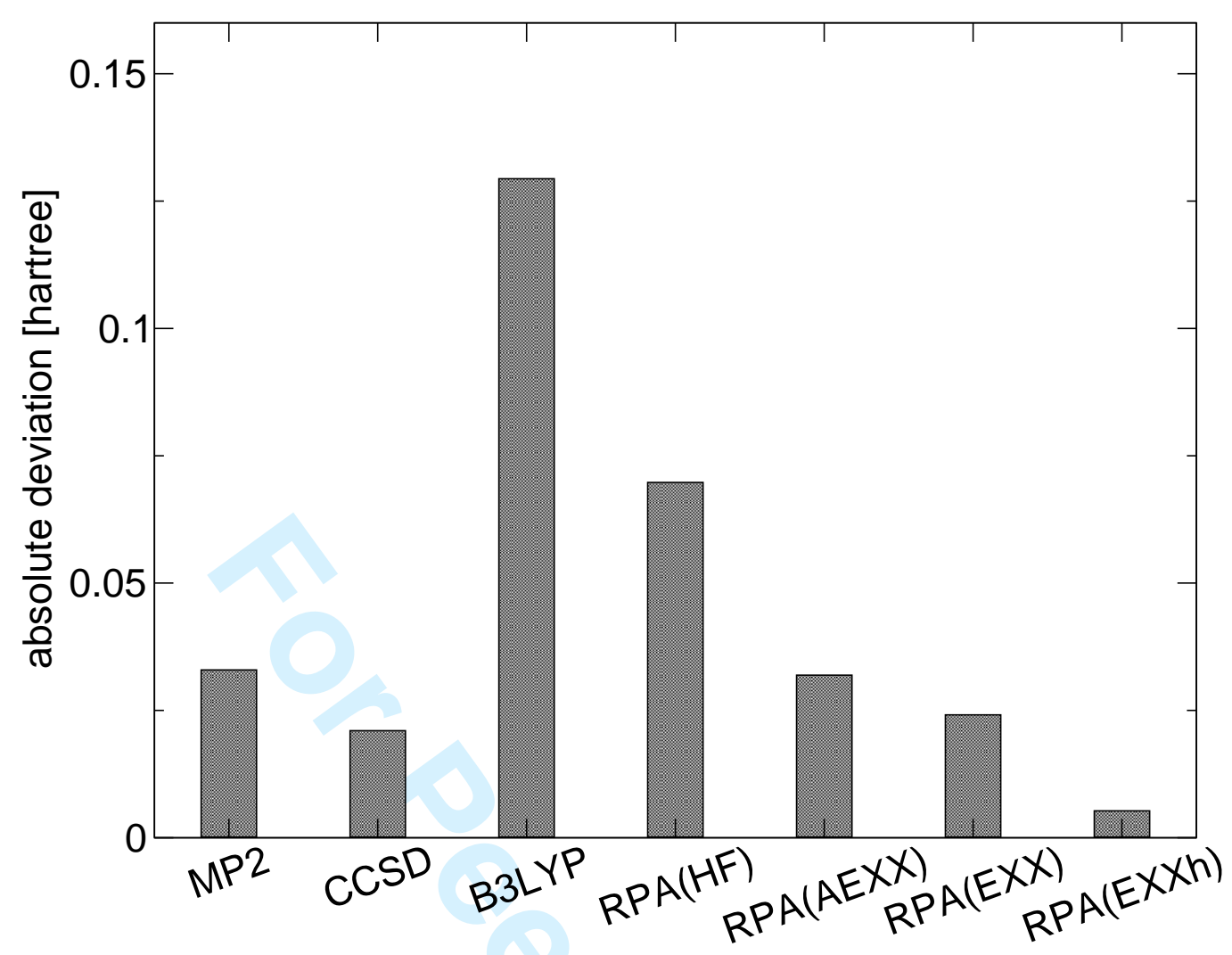

Figure 2. Mean absolute deviations from $\operatorname{CCSD}(\mathrm{T})$ correlation energies (defined in all cases as difference of the total energy with the HF total energy) of 21 molecules, aug-cc-pVTZ basis set. 


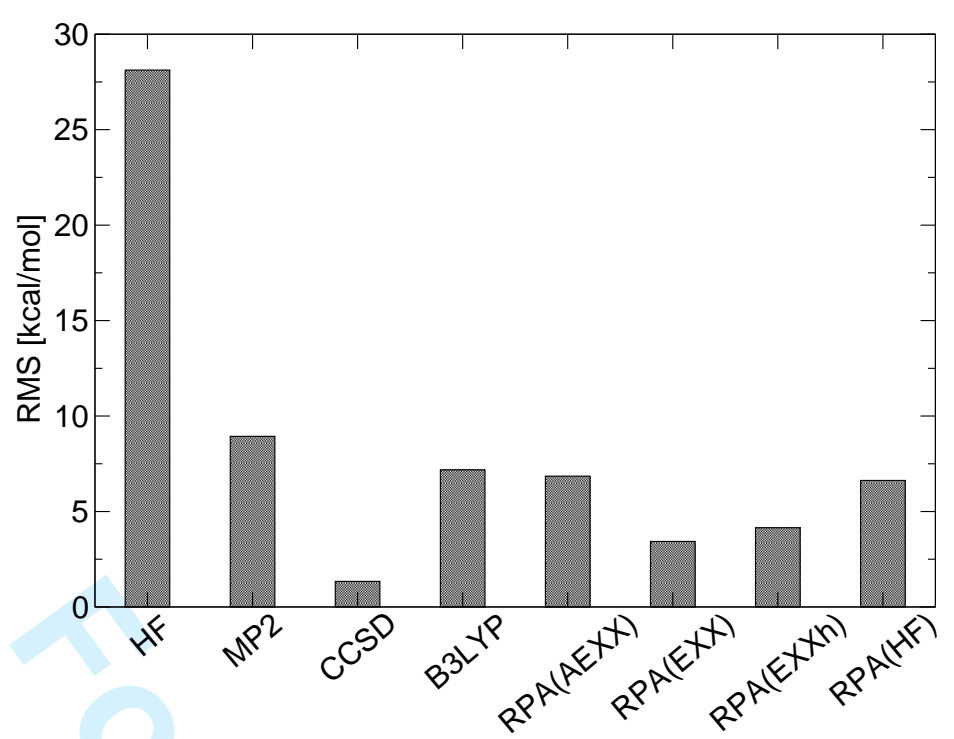

(a)

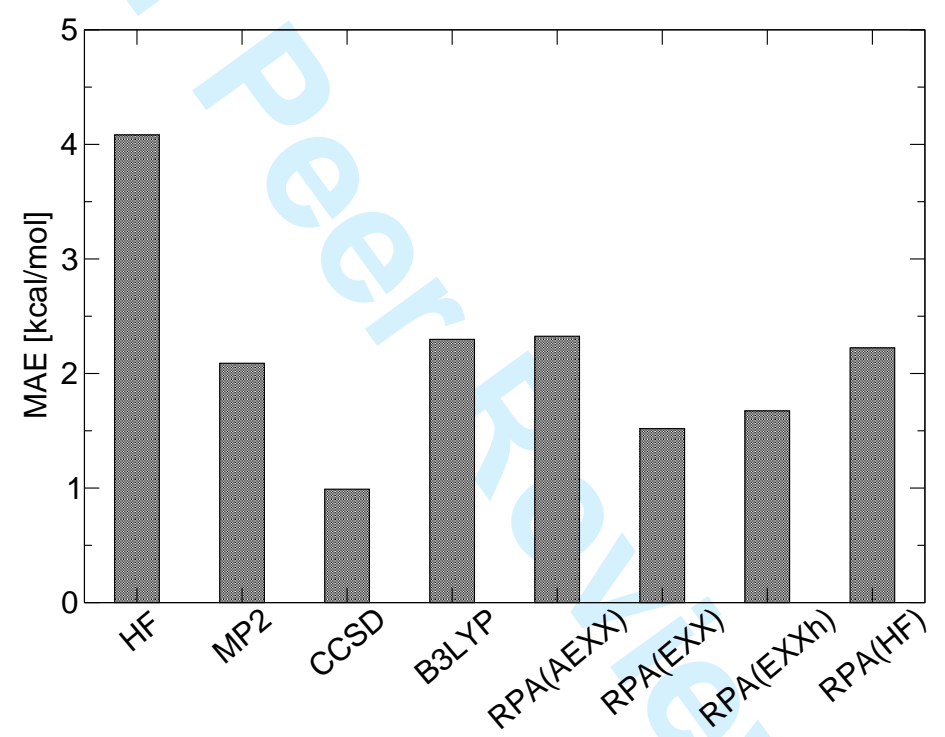

(b)

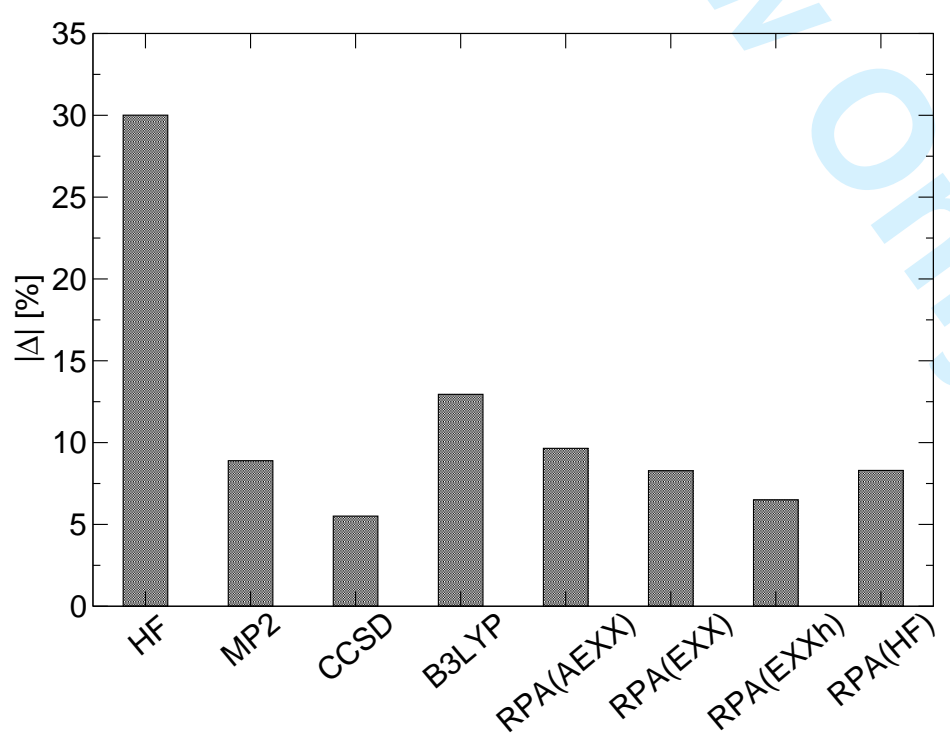

(c)

Figure 3. Root mean squared errors (RMS), mean absolute errors (MAE) and total relative deviations $(|\Delta|)$ from $\operatorname{CCSD}(\mathrm{T})$ reference values for the 14 reactions of table 2. 


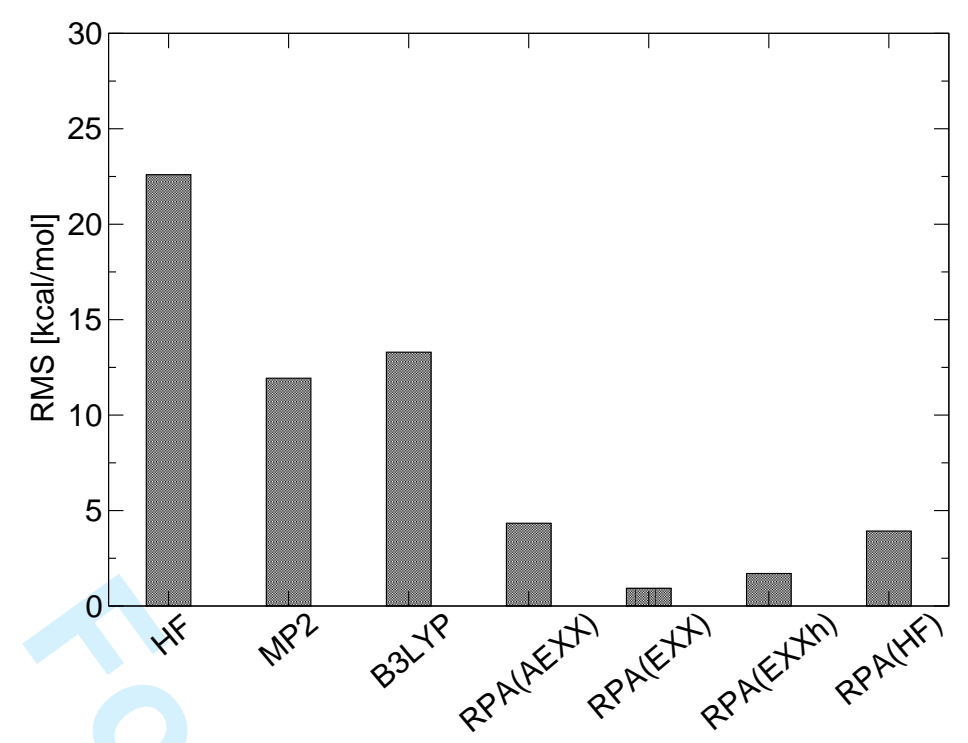

(a)

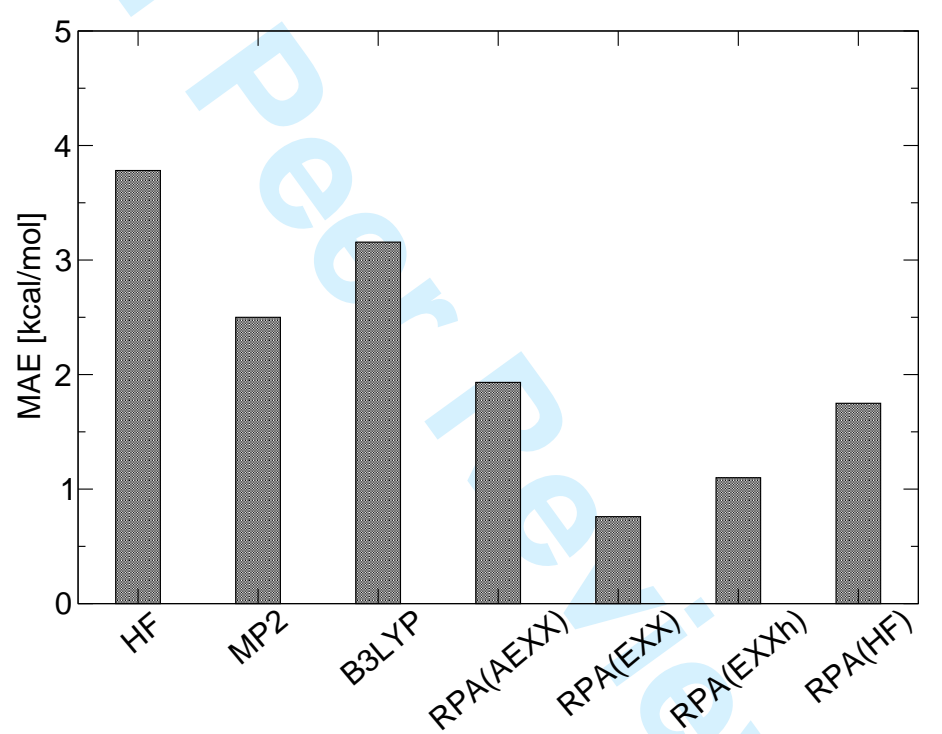

(b)

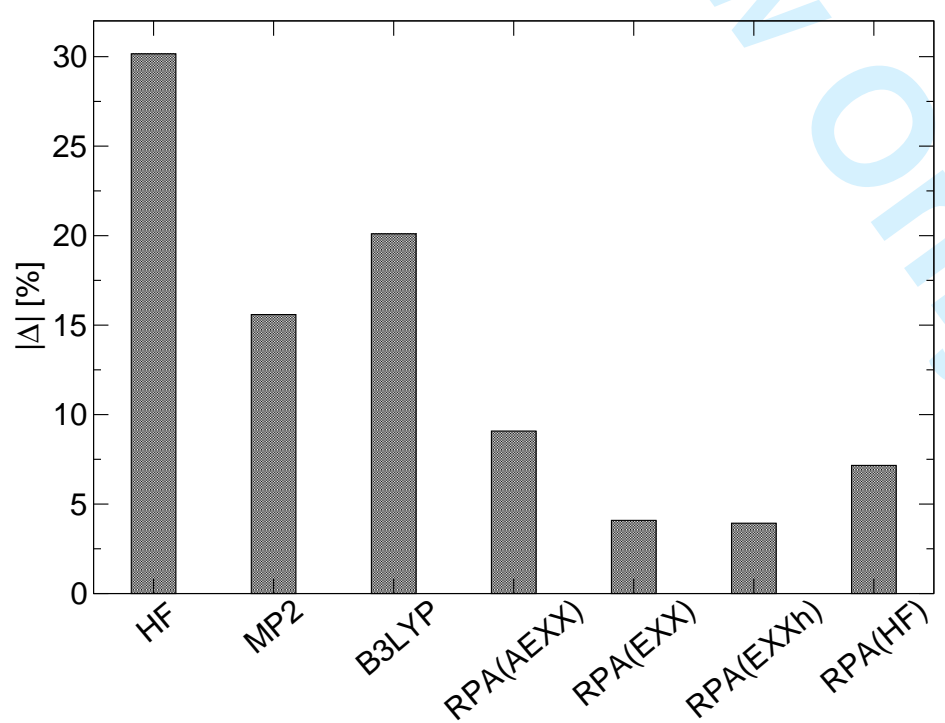

(c)

Figure 4. Root mean squared errors (RMS), mean absolute errors (MAE) and total relative deviations $(|\Delta|)$ from CCSD reference values for the 14 reactions of table 2. 